\title{
Correction to: Maternal HBsAg carriers and pregnancy outcomes: a retrospective cohort analysis of 85,190 pregnancies
}

Yulong Zhang ${ }^{\dagger}$, Jiacheng Chen ${ }^{\dagger}$, Tingting Liao, Siwen Chen, Jianying Yan ${ }^{*}$ (I) and Xiaoqian Lin

\section{Correction to: BMC Pregnancy Childbirth 20, 724 (2020) \\ https://doi.org/10.1186/s12884-020-03257-4}

Following publication of the original article [1], the authors reported an error in Tables 1, 2 and 3 headers. The correct tables are given below.

Statement "YLZ and JCC contributed equally to this work." in the Authors' contributions section deleted. "Yulong Zhang and Jiacheng Chen contributed equally to this work." was inserted as article note.

The original article [1] has been updated.

Published online: 12 February 2021

\section{Reference}

1. Zhang Y, Chen J, Liao T, et al. Maternal HBsAg carriers and pregnancy

outcomes: a retrospective cohort analysis of 85,190 pregnancies. BMC

Pregnancy Childbirth. 2020;20:724 https://doi.org/10.1186/s12884-020-03257-4.

The original article can be found online at https://doi.org/10.1186/s12884020-03257-4

*Correspondence: 505633435@qq.com

${ }^{\dagger}$ Yulong Zhang and Jiacheng Chen contributed equally to this work.

Department of Obstetrics and Gynecology, Fujian Provincial Maternity and Children's Hospital, Affiliated Hospital of Fujian Medical University, Fuzhou

350001, Fujian, China

C C The Author(s). 2021 Open Access This article is licensed under a Creative Commons Attribution 4.0 International License, which permits use, sharing, adaptation, distribution and reproduction in any medium or format, as long as you give appropriate credit to the original author(s) and the source, provide a link to the Creative Commons licence, and indicate if changes were made. The images or other third party material in this article are included in the article's Creative Commons licence, unless indicated otherwise in a credit line to the material. If material is not included in the article's Creative Commons licence and your intended use is not permitted by statutory regulation or exceeds the permitted use, you will need to obtain permission directly from the copyright holder. To view a copy of this licence, visit http://creativecommons.org/licenses/by/4.0/. The Creative Commons Public Domain Dedication waiver (http://creativecommons.org/publicdomain/zero/1.0/) applies to the data made available in this article, unless otherwise stated in a credit line to the data. 
Table 1 Baseline characteristics of the included patients ${ }^{a}$

\begin{tabular}{|c|c|c|c|c|}
\hline & $\begin{array}{l}\text { HBV-positive } \\
(n=9699)\end{array}$ & $\begin{array}{l}\text { HBV-negative } \\
(n=73,076)\end{array}$ & $P$ value & OR \\
\hline Maternal age (yrs, mean \pm SD) & $30.33 \pm 4.50$ & $30.28 \pm 4.45$ & $P=0.30$ & \\
\hline Gestational age (weeks, mean \pm SD) & $38.18 \pm 2.96$ & $38.17 \pm 3.51$ & $P=0.76$ & \\
\hline \multicolumn{5}{|l|}{ Gravidity } \\
\hline 1 & $3323(34.26 \%)$ & $28,076(38.42 \%)$ & $P<0.001$ & $0.84(0.80,0.87)$ \\
\hline$>1$ & $6376(65.74 \%)$ & $45,000(61.58 \%)$ & $P<0.001$ & $1.20(1.15,1.25)$ \\
\hline \multicolumn{5}{|l|}{ Parity } \\
\hline 1 & $82(0.85 \%)$ & $760(1.04 \%)$ & $P=0.07$ & $0.81(0.65,1.02)$ \\
\hline$>1$ & $9617(99.15 \%)$ & $72,316(98.96 \%)$ & $P=0.07$ & $1.23(0.98,1.55)$ \\
\hline \multicolumn{5}{|l|}{ Mode of delivery } \\
\hline Instrumental vaginal & $1903(19.62 \%)$ & $14,009(19.17 \%)$ & $P=0.29$ & $1.03(0.98,1.09)$ \\
\hline Cesarean section & $3380(34.85 \%)$ & $24,729(33.84 \%)$ & $P=0.05$ & $1.05(1.00,1.09)$ \\
\hline Vaginal delivery & $4373(45.09 \%)$ & $33,907(46.40 \%)$ & $P=0.01$ & $0.95(0.91,0.99)$ \\
\hline Regional analgesia & $43(0.44 \%)$ & $431(0.59 \%)$ & $P=0.07$ & $0.75(0.55,1.03)$ \\
\hline \multicolumn{5}{|l|}{ Birth weight(g) } \\
\hline$<3000$ & $2855(29.44 \%)$ & $20,571(28.15 \%)$ & $P=0.008$ & $1.06(1.02,1.122)$ \\
\hline 3000-3499 & $4026(41.51 \%)$ & $30,721(42.04 \%)$ & $P=0.32$ & $0.98(0.94,1.02)$ \\
\hline 3500-3999 & 2231 (23.00\%) & 17,085 (23.38\%) & $P=0.41$ & $0.98(0.93,1.03)$ \\
\hline$\geq 4000$ & 388 (6.05\%) & $2718(6.43 \%)$ & $P=0.17$ & $1.08(0.97,1.20)$ \\
\hline
\end{tabular}

Table 2 The association between HBV positive pregnancies and outcomes

\begin{tabular}{lllll}
\hline & $\begin{array}{l}\text { HBV-positive } \\
(\boldsymbol{n}=\mathbf{9 6 9 9 )}\end{array}$ & $\begin{array}{l}\text { HBV-negative } \\
(\boldsymbol{n}=\mathbf{7 3 , 0 7 6 )}\end{array}$ & OR value & \\
\hline Gestational hypertension & $157(1.62 \%)$ & $1421(1.94 \%)$ & $P=0.028$ & $0.83(0.70,0.98)$ \\
Preeclampsia & $139(1.43 \%)$ & $1302(1.78 \%)$ & $P=0.014$ & $0.80(0.67,0.96)$ \\
HELLP syndrome & $5(0.05 \%)$ & $48(0.07 \%)$ & $P=0.605$ & $0.78(0.31,1.97)$ \\
PPROM & $2577(26.57 \%)$ & $20,776(28.43 \%)$ & $P<0.001$ & $0.91(0.87,0.96)$ \\
Postpartum hemorrhage & $218(2.25 \%)$ & $1424(1.95 \%)$ & $P=0.047$ & $1.16(1.00,1.34)$ \\
GDM & $1663(17.15 \%)$ & $11,982(16.40 \%)$ & $P=0.062$ & $1.06(1.00,1.12)$ \\
ICP & $149(1.54 \%)$ & $334(0.46 \%)$ & $P=0.001$ & $3.40(2.80,4.13)$ \\
placental abruption & $140(1.44 \%)$ & $905(1.24 \%)$ & $P=0.089$ & $1.17(0.98,1.40)$ \\
Premature birth & $1158(11.94 \%)$ & $8424(11.53 \%)$ & $P=0.234$ & $1.04(0.97,1.11)$ \\
Small for gestational age & $392(0.22 \%)$ & $3165(0.19 \%)$ & $P=0.192$ & $0.93(0.84,1.04)$ \\
\hline
\end{tabular}


Table 3 Gestational complications in Vaginal delivery

\begin{tabular}{lllll}
\hline & $\begin{array}{l}\text { HBV-positive } \\
(\boldsymbol{n}=\mathbf{6 4 4 7 )}\end{array}$ & $\begin{array}{l}\text { HBV-negative } \\
(\boldsymbol{n}=\mathbf{4 9 , 2 0 6})\end{array}$ & $\boldsymbol{P}$ & OR \\
\hline Gestational hypertension & $88(1.30 \%)$ & $804(1.41 \%)$ & $P=0.106$ & $0.83(0.67,1.04)$ \\
Preeclampsia & $49(0.76 \%)$ & $479(0.97 \%)$ & $P=0.096$ & $0.78(0.58,1.05)$ \\
HELLP syndrome & $1(0.02 \%)$ & $6(0.01 \%)$ & $P=0.851$ & $1.27(0.15,10.57)$ \\
PPROM & $2018(31.30 \%)$ & $16,808(34.16 \%)$ & $P<0.001$ & $0.88(0.83,0.93)$ \\
Postpartum hemorrhage & $178(2.76 \%)$ & $1151(2.34 \%)$ & $P=0.037$ & $1.19(1.01,1.39)$ \\
GDM & $1079(16.74 \%)$ & $7811(15.87 \%)$ & $P=0.076$ & $1.07(0.99,1.14)$ \\
ICP & $78(1.21 \%)$ & $161(0.33 \%)$ & $P<0.001$ & $3.73(2.84,4.90)$ \\
Placental abruption & $101(1.57 \%)$ & $537(1.09 \%)$ & $P=0.001$ & $1.44(1.16,1.79)$ \\
Premature birth & $669(10.38 \%)$ & $4546(9.24 \%)$ & $P=0.096$ & $1.14(1.04,1.24)$ \\
Small for gestational age & $265(4.11 \%)$ & $1817(3.69 \%)$ & & $1.12(0.98,1.28)$ \\
\hline
\end{tabular}

